\title{
Commentary Breast cancer research and the European Union Clinical Trials Directive
}

\author{
Norman Williams
}

Clinical Trials Group of the Department of Surgery, Royal Free and University College Medical School, University College London, London W1W 7EJ, UK

Corresponding author: Norman Williams (e-mail: n.williams@ctg.ucl.ac.uk)

Published: 20 April 2004

Breast Cancer Res 2004, 6:145-147 (DOI 10.1186/bcr800)

(C) 2004 BioMed Central Ltd

\begin{abstract}
Running clinical trials in the commercial sector has always been associated with a certain amount of bureaucracy due to the stringent requirements needed to bring a new drug onto the market. Noncommercial trials have largely been performed outside these requirements. New legislation brought about as a result of the implementation of the European Union Clinical Trials Directive will change this two-tiered approach by harmonizing regulations in all member states. Those who run noncommercial clinical trials will have to find cost-effective ways of dealing with this legislation if such work is to continue in Europe.
\end{abstract}

Keywords: clinical trial, European Union, legislation, government, regulation

Performing clinical trials can be frustrating at times, and one of the most exasperating tasks is corresponding with local research ethics committees. We recently quantified the delays in responses regarding a protocol amendment, and found that a substantial proportion of the centres we dealt with took more than 60 days to approve the amendment; some took considerably longer [1]. Staff in our trials centre have to 'chase' this information, which means that they have less time for other tasks; furthermore, a delay in approval meant a delay to the implementation of the protocol amendment in those local centres, which is far from satisfactory.

It was therefore very pleasing to hear that a forthcoming European Union (EU) directive included a section specifically stating that decisions by ethics committees should be given in less than 60 days for new protocols and 35 days for protocol amendments. Our joy was shortlived, however, when we realized that other aspects of the directive might actually increase our workload.

The EU produces many directives - it is the mechanism by which decisions made centrally can be implemented in the member states. Directives cover a wide range of human activity, as can be gleaned from their titles: Food Supplements, Software Patents, Data Protection, Direct Marketing, Molecular Farming, Traditional Herbal Medicinal Products and Mutual Recognition of Professional Qualifications, to name but a few. The directive that sets a time limit on research ethics committees is Directive 2001/20/EC [2], entitled the Clinical Trials Directive (CTD), which aims to harmonize the administration of clinical trials throughout the EU. Member states are required to implement the CTD into national legislation by 1 May 2004 (which, incidentally, is the same day that the EU will grow from 15 to 25 member states).

The CTD itself is a fairly short and general document, and on first reading it appears to be perfectly logical. It is during the transposition of the CTD into domestic law where the devilish details emerge, because the passing of one law can have knock-on effects on many others. The CTD has a wide scope, and covers many aspects of the conduct of clinical trials in the EU on human subjects (including healthy volunteers participating in phase I studies) that involve a medicinal product. Ethics 
committees will be established on a legal basis, and legal status will be provided for certain procedures. There will also be legislation to cover the manufacture, import, labelling and quality assurance of investigational medicinal products; the creation of a European database of all clinical trials conducted in the EU; and the provision for safety monitoring of patients in trials (pharmacovigilance), including procedures for recording and reporting adverse drug reactions on another pan-European database. Adherence to both Good Clinical Practice (GCP) and Good Manufacturing Practice will be 'policed'; in the UK the Medicines and Healthcare products Regulatory Agency will begin a statutory inspection programme of GCP compliance. The Medicines and Healthcare products Regulatory Agency (home page: http://www.mhra.gov.uk) has been designated as the primary source of authoritative advice and information on the CTD and associated legislation.

The basic aims of the CTD are to protect participants in clinical trials and to ensure that data generated from these trials are accurate and verifiable, which are important in all trials. So what is the problem? There are several, but one of the larger problems is that the CTD does not distinguish between commercial and noncommercial clinical trials that is, between trials that are run primarily to obtain a marketing authorization for a drug and trials that are run primarily to answer an important scientific question.

For those of us who have experience of industrysponsored clinical trials, the principles of the CTD seem very sensible; indeed, little of it is truly novel. However, there seems to be an assumption that industry is using the best model, and this is not always justified. Take, for example, the concerns about the practical application of GCP. Those who have experience of trials sponsored by the pharmaceutical industry are often frustrated by the attention to seemingly mindless trivia, and the well meaning but misguided bureaucracy [3]. Some of the other difficult areas include the introduction of a commercial-style approach to trial monitoring and pharmacovigilance that may not always be appropriate, particularly where the trial is testing a product that is already on the market.

Perhaps the academic model is the best model to use. However, can we honestly say that all academic run clinical trials have been run to the highest standards? Are we certain that every trial has been run to protocol, that the studies have been adequately powered, that all patients have truly given 'informed consent', and that appropriate mechanisms have always been in place to deal with the reporting of adverse events? Are we not aware of questionably run trials whose primary aim seems to be the generation of a publication for the advancement
In spite of these problems, let no one forget the important contributions that have been made by noncommercial trials. This is perhaps best illustrated by those trials concerned with breast cancer, the results of which have saved literally millions of lives, notably trials investigating the role of tamoxifen in early breast cancer. Special issues that typify these studies include comparisons of the risks and benefits of similar interventions (shorter or longer duration of tamoxifen), investigations of long-term outcomes $(5,10,20$ years after cessation of therapy), finding new roles for old drugs, determining efficacy in realistic settings (pragmatic trials), and comparisons with nondrug therapy (e.g. surgery and radiotherapy). Such studies answered very important questions without including a comparison with a novel drug; few of these studies would have been sponsored by the pharmaceutical industry.

Indeed, it is the role and definition of 'sponsor' that is causing the most angst for those who run noncommercial trials. The CTD requires a sponsor to take responsibility for the initiation, management and/or financing of a trial, which for commercial trials would normally be a drug company. In noncommercial trials (particularly multicentre studies) these responsibilities are often distributed among many individuals and organizations. For example, the trial might have been initiated in several centres, managed by a steering committee and data safety and monitoring committee, with funding provided by two or three sources. Many of the institutions currently involved (universities, hospitals and funding bodies) have categorically stated that they are unwilling to take on the responsibility of a sponsor, particularly for centres over which they have no control. Even institutions that are currently running single centre studies are unsure of what they will be letting themselves in for and so have declined to take on the role of sponsor. Perhaps a compromise could be reached that allowed for shared responsibility, or a special agency could be set up at a national (or even European) level to act nominally as sponsor.

There is a worry that, in order to deal with issues of sponsorship, pharmacovigilance, and other factors, noncommercial trials will now require funding to a scale that approaches that of commercial trials. In industry costs can be passed on, but this is not an option for noncommercial trials. So who will pay - the government (unlikely) or industry? Although there are precedents for industry-academia collaborative ventures (e.g. ATAC [Arimidex, Tamoxifen Alone or in Combination], a trial of a novel adjuvant hormonal therapy in early breast cancer $[4,5])$, such examples are few and far between, particularly if the commercial benefits are small. It seems that the most likely source of this extra resource will be grant-funding bodies such as the charitable organizations. Already overstretched budgets will have to be stretched further to meet the new legislative requirements. This will ultimately 
mean that we will see fewer of the smaller 'pilot' studies and the trend will be toward larger, multicentred trials, but fewer of them.

Another concern is that the legislation brought about by the CTD will focus the 'mindset' of the various agencies concerned on clinical trials of medicinal products, to the detriment of work that falls outside of this definition. Will a regulatory agency require the name of a sponsor for a study setting up a tumour bank to investigate translational work? How closely will an ethics committee look at an application performing dietary studies on healthy volunteers? Would the cranberry juice used in a trial to investigate its effects on urinary tract infections have to pass the stringent requirements of Good Manufacturing Practice, and will there be an expectation that adverse events will be reported?

The CTD has been 'in the pipeline' for more than 3 years. Unfortunately, many academic clinical trialists across Europe have only recently woken up to the changes that the CTD will bring, which had to come sooner or later. For better or for worse, on Saturday 1 May 2004 the rules will alter significantly. The challenge we all face is to find innovative ways of dealing with these changes, or the future of clinical trial research in Europe will be bleak.

\section{Competing interests}

None declared.

\section{References}

1. Williams NR, McRae K, Houghton J: Delays in ethics committee approval: an English perspective [abstract]. Breast Cancer Res Treat 2003, 82(suppl 1):S80.

2. The European Parliament and the Council of the European Union: Directive 2001/20/EC of the European Parliament and of the Council of 4 April 2001 on the approximation of the laws, regulations and administrative provisions of the Member States relating to the implementation of good clinical practice in the conduct of clinical trials on medicinal products for human use. Official J Eur Communities 2001, L121:34-44.

3. Baum M, Buchanan M, Baselga J, Cataliotti L, Jassem J, Piccart $\mathrm{M}$ : The future of breast cancer research in danger. Eur $J$ Cancer 2002, 38:2210-2213.

4. The ATAC (Arimidex, Tamoxifen Alone or in Combination) Trialists' Group: Anastrozole alone or in combination with tamoxifen versus tamoxifen alone for adjuvant treatment of postmenopausal women with early breast cancer: first results of the ATAC randomised trial. Lancet 2002, 359:2131-2139.

5. Baum M: Background. In History and Advancement of Anastrozole in the Treatment of Breast Cancer. Edited by Buzdar A, Baum M. London: The Royal Society of Medicine Press; 2003:3-6. 